\title{
A Study of Extemal Nasal Splints used in Nasal Bone Fractures and Rhinoplasties
}

\author{
${ }^{1}$ Sudhir M Naik, ${ }^{2}$ Sarika Sudhir Naik \\ ${ }^{1}$ Assistant P rofessor, Department of ENT and Head and Neck Surgery, KVG Medical College and Hospital, Sullia, Karnataka, India \\ ${ }^{2}$ Senior Resident, Department of Anesthesia, KVG Medical College and Hospital, Sullia, Karnataka, India
}

Correspondence: Sudhir M Naik, Assistant Professor, Department of ENT and Head and Neck Surgery, KVG Medical College and Hospital, Kurunjibag-574327, Sullia, DK, Karnataka, India, P hone: 09916807109, e-mail: sud223@ gmail.com

\begin{abstract}
Background/Objectives: Plaster of Paris (POP), thermoplastic splints and self-adhesive padded aluminium splints are the most common splinting methods used after reduction of fractured nasal bones and rhinoplasty. All these methods have their proponents but may have one or more disadvantages in the way of being cumbersome, time-consuming, bulky, conspicuous and expensive.

Design: A retrospective study at KVG Medical College and Hospital, department of ENT and head and neck surgery.

Intervention: 94 cases of splinting done for nasal bone fractures and rhinoplasties were included in our study. POP and adhesive aluminium splints were used to stabilize the nasal framework.

Results: Eleven cases of nondislocated nasal fractures and 10 cases of internal augmentation rhinoplasties were stabilized by aluminium nasal splints. 69 cases of displaced nasal fractures and 4 cases of external rhinoplasties were stabilized by POP splints.

Conclusions: POP splints give the best stabilization for nasal bone fractures as well as for rhinoplasties but are bulky and conspicuous. Aluminium nasal splints are not bulky and conspicuous but cannot be used for fractures with lacerations and external rhinoplasties.

Keywords: Plaster of Paris splint, Aluminium splints, Nasal bone fractures, R hinoplasty.
\end{abstract}

\section{INTRODUCTION}

An external dressing is often used to provide stability to the bony nasal framew ork following the reduction of nasal bone fractures and rhinoplasties. ${ }^{1}$ The external dressing keeps the nasal bridge in alignment for one to two weeks, while the fractured bones set in place. ${ }^{1}$

Common splinting methods include the use of Plaster of Paris (POP), thermoplastic splints, ${ }^{2}$ self-adhesive padded aluminium splints ${ }^{3}$ and many other designs. ${ }^{4,5}$ All these methods have their proponents but may have one or more disadvantages in the way of being cumbersome, timeconsuming, bulky, conspicuous and expensive. ${ }^{6}$

F ractures of the nasal bones are usually a result of trauma and is suggested by external nasal deformity, crepitus or palpable mobile bony segments (Fig. 1). ${ }^{7}$ Pain, swelling and epistaxis are common symptoms and these may be accompanied by ecchymosis of the periorbital soft tissues. ${ }^{7}$ $\mathrm{N}$ asal obstruction is seen if the septum has been displaced. ${ }^{7}$ $\mathrm{Nasal}$ fractures are most common fractures of the facial skeleton and accounts for 40 to $50 \%$ of all the facial fractures. ${ }^{7}$ The incidence is more compared to that in females. ${ }^{8-10}$ The incidence is more in the 2 nd and $3 \mathrm{rd}$ decade. ${ }^{8-10}$ M ost common etiologic factors in adults are assaults, sports injuries and less commonly road traffic accidents (Fig. 2). ${ }^{8-10}$

The nasal bones are flat, rectangular and thinner at the caudal end. ${ }^{11}$ They project from the frontal process of the maxilla and articulate with the upper lateral cartilages and nasion (Fig. 3). ${ }^{11}$ Nearly $80 \%$ of the nasal fractures occur at transition zone between the thicker proximal and thinner distal fragments, which corresponds to lower $1 / 3$ to $1 / 2$ of nasal bones. ${ }^{11}$ The goals of nasal fracture reduction and splinting should be to achieve a cosmetic result similar to the preinjury appearance, and to obtain good nasal airway patency. ${ }^{12}$

$\mathrm{N}$ asal fracture reduction and splinting may be performed under local anesthesia with or without sedation or general anesthesia. ${ }^{13}$ The target of treatment in nasal bone fracture is to restore the appearance and function of the nose to their pretrauma state. ${ }^{13}$ It is generally accepted that the final result of treatment cannot be properly evaluated until 1 or 2 years have passed since treatment. ${ }^{13}$ The reason is that both the trauma and the reduction might cause fibrosis that can lead to a secondary deformity of the nasal pyramid. ${ }^{13}$ In rhinoplasties, splinting is done to fix the nose to a desired cosmetic appearance. $^{13}$ 


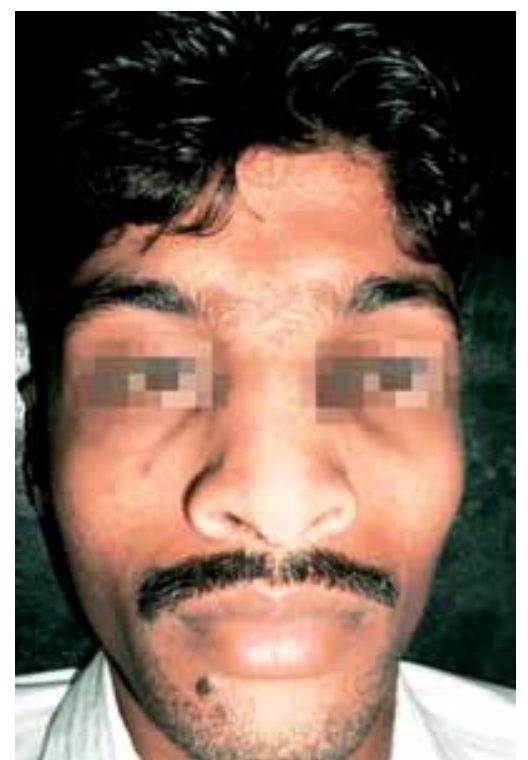

Fig. 1: Nasal fracture with swelling of nasal bridge

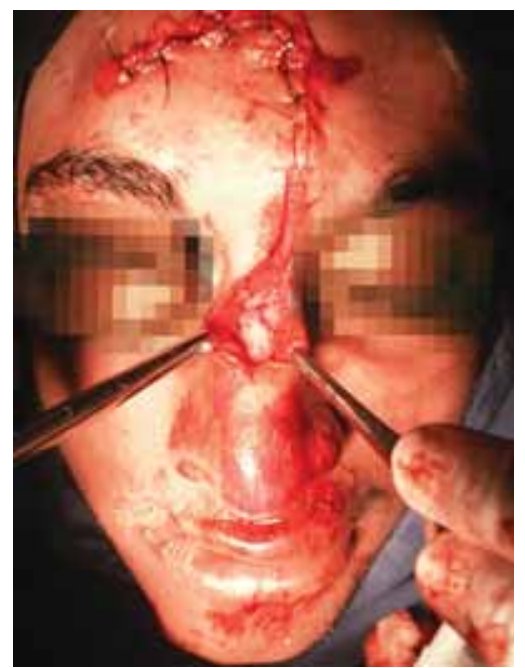

Fig. 2: Compound nasal fracture with displacement

We describe two splinting methods for nasal fractures and rhinoplasty, which is simple to use, inexpensive, well accepted and tolerated by patients. We used POP and custom made aluminium splints, which are easy to trim and tailor to individual patient's requirements.

\section{MATERIALS AND METHODS}

This is a retrospective study done in department of ENT KV G M edical College and Hospital, Sullia. A total of 94 cases who had undergone reduction of fractured nasal bones and rhinoplasty were included under the study. In 73 cases, POP splints were used and in the rest 21 cases self-adhesive padded aluminium splints were used.

In our study, 71 (75.5\%) were males and $23(24.5 \%)$ were females. In our study the youngest patient was 8 years

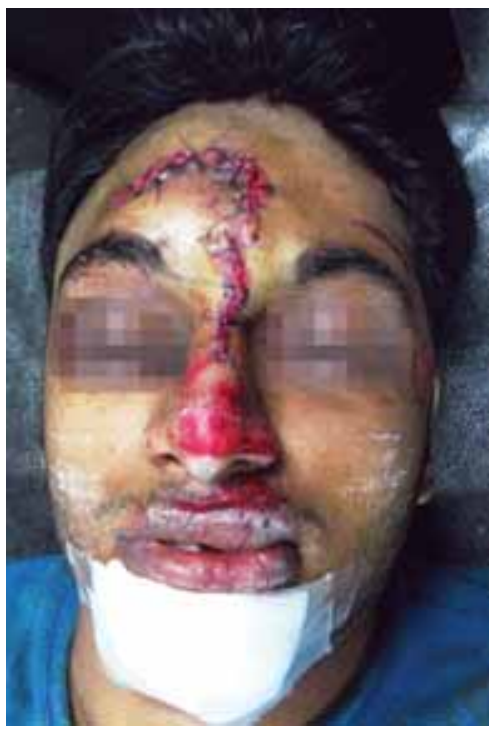

Fig. 3: Compound nasal fracture reduced and wound repaired

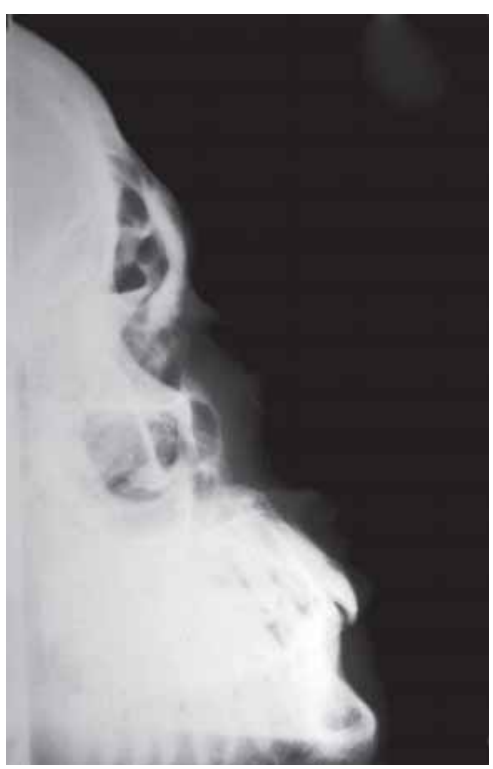

Fig. 4: X-ray lateral view showing fracture

old and the oldest was 54 years old. Their mean age was 24 years.

For patients with nasal fractures a detailed history, including information on the mode of injury, any previous injury or previous nasal deformity, and the presence of posttrauma airway obstruction were recorded in detail. A previously taken recent photograph of the patient was examined.

A II the patients posted for rhinoplasty had preoperative photographs taken and the expected results explained to the patients. All patients underwent a thorough external nasal and anterior rhinoscopic examination. All the nasal fractures had X-ray waters (occipitomental) and lateral views of the nasal bones and paranasal sinuses taken (Fig. 4). 
CT scan with axial and coronal cuts of the nasal bones and the paranasal air sinuses were examined (Figs 5 and 6).

Eleven patients had nondisplaced nasal fractures, so aluminium splints were used in them. Ten patients had augmentation internal rhinoplasties, and so, simple aluminium splints were used in them. 69 patients had displaced nasal fractures and also among them 17 had external wounds with compound fractures, and hence after reduction and skin wound repair POP splint was used. Four patients underwent external rhinoplasty, and so, POP cast was used in them.

When edema of the nasal pyramid or surrounding tissues was present, reduction was postponed for 3 to 4 days to allow the swelling to subside. Once it did, we used photographs of the patient taken before the injury to compare the state of the nose pre- and post-trauma in order to decide whether reduction was necessary. Of the 80 cases, in

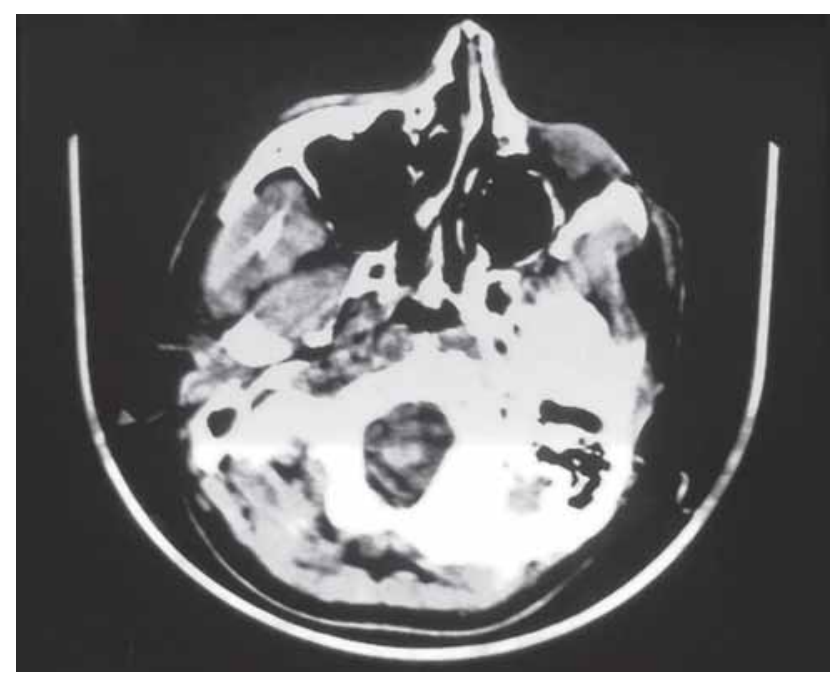

Fig. 5: CT axial view showing fracture of nasal bone and septal dislocation

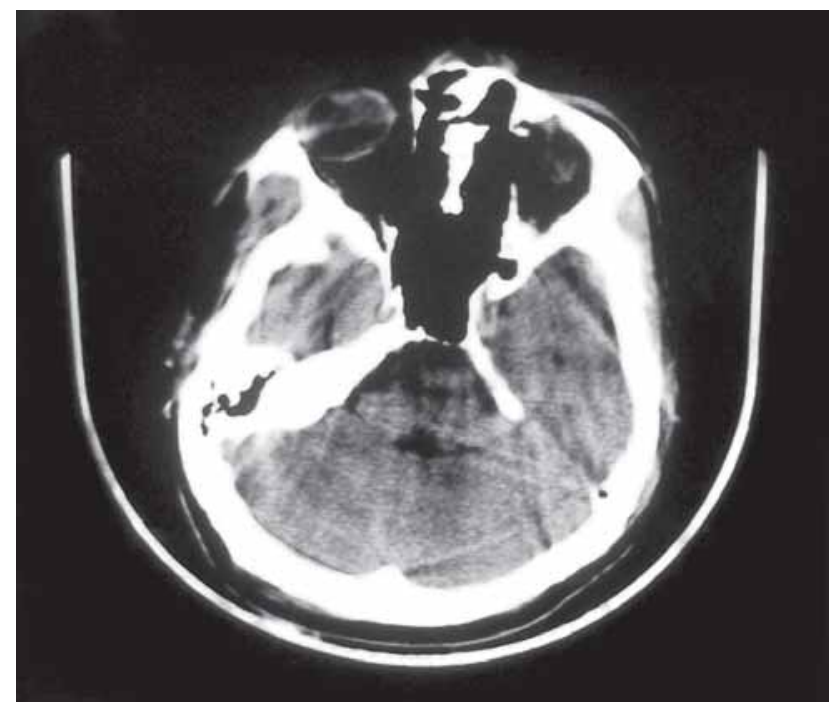

Fig. 6: CT axial view showing fracture of nasal bone
57 cases close reduction was performed within 24 hours, in 14 cases it was done on the 2 nd day, in four cases on the 3rd day, and in five cases on the 4th day.

\section{PROCEDURE}

For nasal fractures, cavities were sprayed with $15 \%$ lidocaine spray and packed with 4\% lidocaine topical anesthesia mixed with xylometazoline $0.1 \%$ soaked cotton strips and left in place for 10 minutes.

In all cases, the infratrochlear, infraorbital and nasopalatine nerves were blocked with a local anesthetic $1 \%$ lidocaine. Reduction was done by exerting external pressure and by using Boies' elevator, Asch's forceps (Fig. 7), and Wal sham's forceps (Fig. 8) depending on the degree of deformity. Nasal packing was done with framycetin ribbons. POP casts were cut in shape as described in the photo (Fig. 9) and applied to the reduced nasal bones and left in place for 21 days (Figs 10 and 11). The splint was worn for 24 hours for the first week and only during night time for the 2nd and 3rd week. General anesthesia was used in 15 patients for nasal fracture reduction. M ost of these patients were younger than 14 years of age. In 69

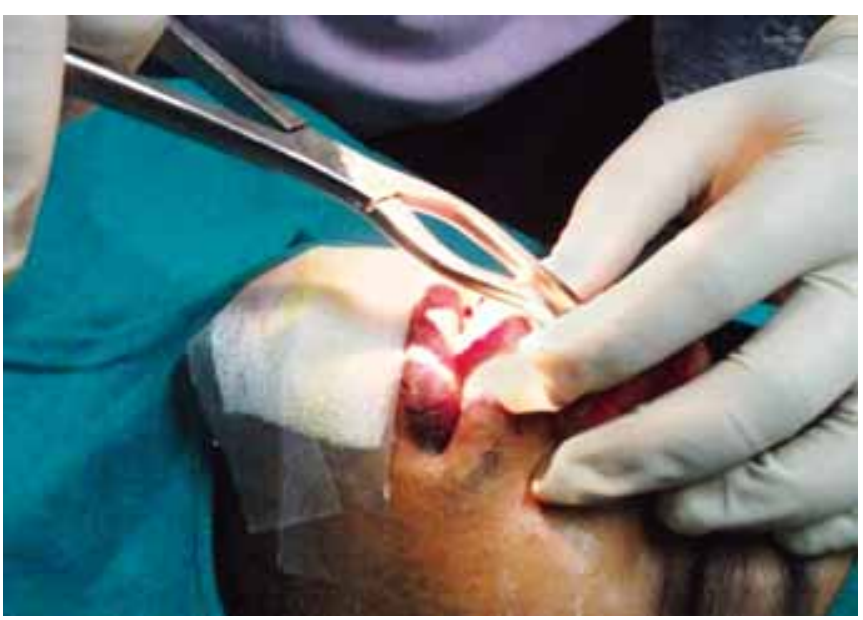

Fig. 7: Asche's forceps used to reduce the septum

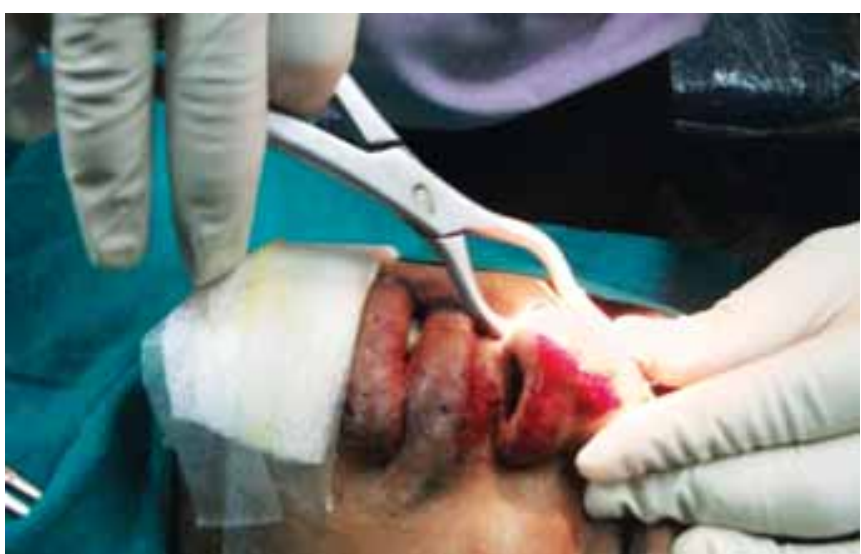

Fig. 8: Walsham forceps used to reduce the lateral wall 


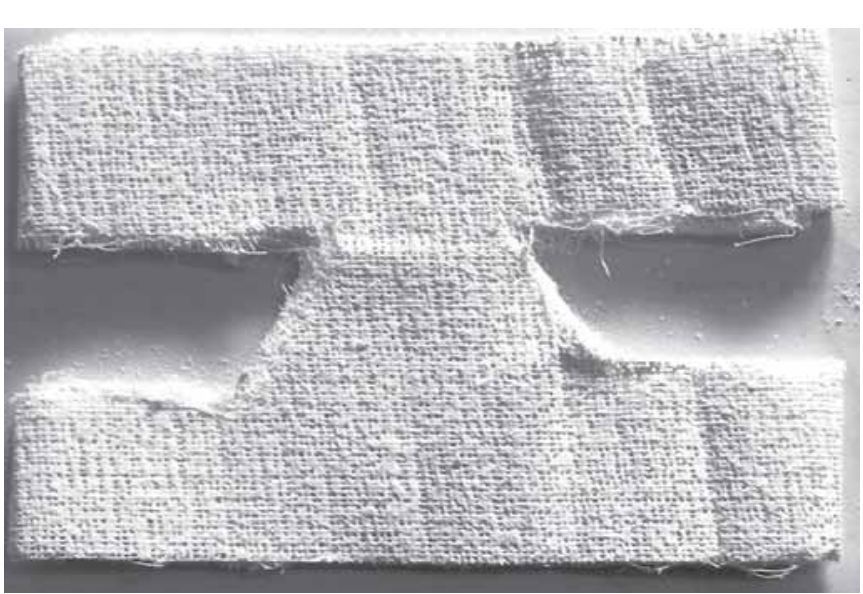

Fig. 9: POP cut for splinting

cases of displaced nasal fractures, POP splints were used and in 11 cases of nondisplaced nasal fractures aluminium splints were used.

Ten cases of internal augmentation rhinoplasties were splinted by aluminium splints and four cases of external rhinoplasties were splinted by POP.

\section{RESULTS}

All the 94 patients had nasal packing with nasal tampon or ribbon packs, which were removed after 48 hours. The POP splints were left in place for 21 days and aluminium splints for 14 to 20 days.

Local anesthesia was found to be more effective for reducing fractured nasal bones as adjustments with manipulation could be done as per patient's and attendant's advice. Results were obvious after splint removal. The patients were followed up for 3 to 8 months.

73 patients splinted with POP felt it to be bulkier and wanted it to be removed earlier. 21 patients in whom al uminium splints were used remarked the splints were light and the adhesive was irritating the skin (Figs 12 and 13). The costs of both the splints were nearly equal. The procedure of the POP splints was cumbersome, time consuming and conspicuous. The aluminium splints were readymade and easy to use, lightweight and skin colored, so not conspicuous. In 32 patients with POP, the splints were removed after 7 days and replaced by less bulky aluminium splints.

Of the 80 patients who underwent reduction, 77 (95.94\%) expressed complete satisfaction with their clinical outcome. Three patients who were not satisfied were advised rhinoplasty after 6 months. All the 14 cases who had rhinoplasty expressed satisfaction with results.

\section{DISCUSSION}

$\mathrm{N}$ asal splinting after reduction of nasal fracture or rhinoplasty, osteotomies is done to stabilize the nasal

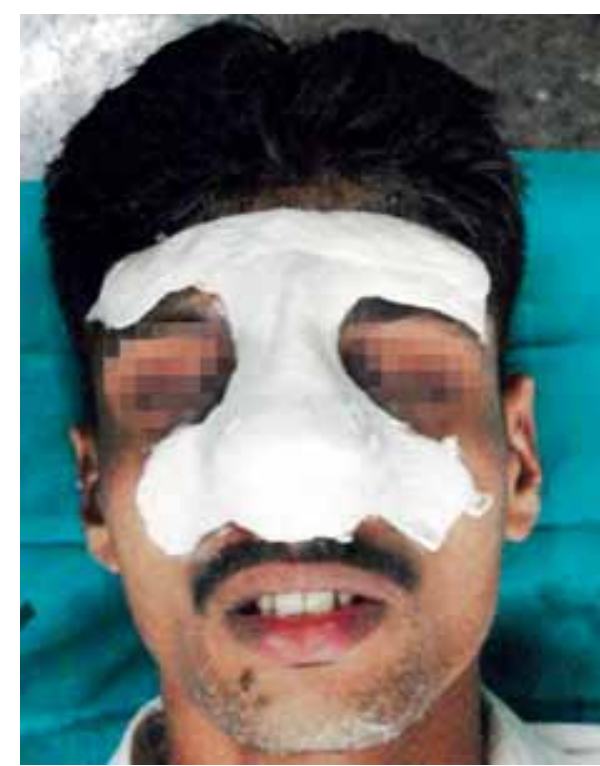

Fig. 10: POP applied after reduction

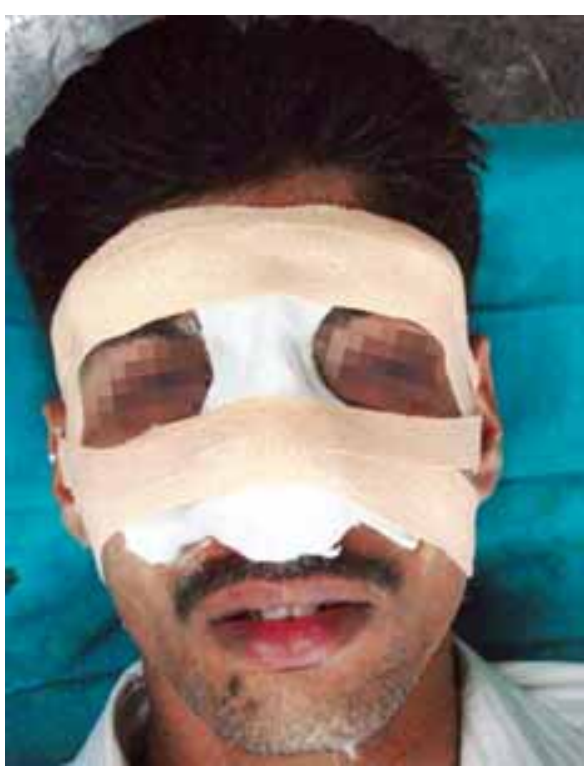

Fig. 11: POP splint fixed

framework. ${ }^{6}$ Common materials include POP, aluminum and heat-malleable plastic splints. ${ }^{6}$ External dressing with POP is commonly used as it is inexpensive, readily available and easy to use. ${ }^{6} \mathrm{H}$ owever, it is a cumbersome, bulky dressing, and requires time before rigidity is obtained. ${ }^{6}$ Dry debris from the plaster can irritate the eyes. ${ }^{6}$

Self-adhesive metal splinting has been used after reduction of nasal fractures and rhinoplasty for many years. ${ }^{6}$ The metal splint used is firm to provide stability and malleable for trimming and folding closely around the nasal bridge. ${ }^{6}$

The double layer of rubber dressing provides good insulation and protection to the skin. ${ }^{6} \mathrm{No}$ complications directly related to the use of this dressing and splint has been noticed. ${ }^{6}$ Self-padded aluminium splints are firm yet thin and fairly inconspicuous dressing, well-tolerated by 


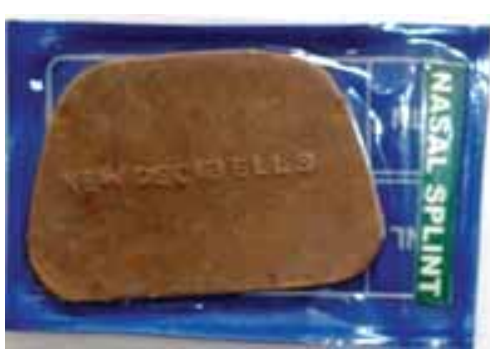

Fig. 12: Self-adhesive aluminium splints

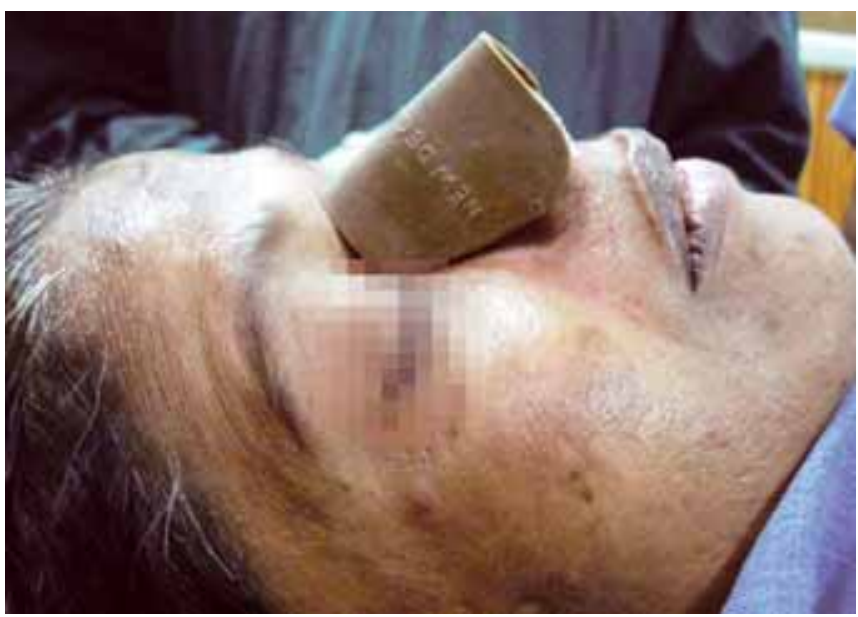

Fig. 13: Aluminium splints applied for rhinoplasty

the patients. ${ }^{6}$ It comes as the skin colored camouflage, which is used with a skin colored dressing rather than white dressing tape. ${ }^{6}$

The nasal bones are the most commonly fractured bones in the facial skeleton. ${ }^{14}$ A ccurate clinical diagnosis and appropriate surgical interventions are very important in the management of nasal fractures. ${ }^{14} \mathrm{~N}$ asal bone fractures are not life-threatening but mismanagement of nasal fractures can lead to both cosmetic and functional deformities. ${ }^{14}$

In our country, nasal bone fracture amounts to grievous hurt, so all these are recorded as medicolegal cases. ${ }^{14}$ So, a thorough history and careful clinical examination are done and recorded. ${ }^{14}$ Photographs are taken at the time of injury, before reduction and after reduction. ${ }^{14} \mathrm{X}$-rays of the nasal bones lateral views and $X$-ray PNS are supplementary in diagnosis. Sometimes, the fractures are associated with significant edema, and so they cannot be accurately reduced at that time. ${ }^{14}$ Only if the edema is present then reduction should be delayed till edema subsides. ${ }^{14}$ Indications for immediate reduction even in edema are grossly displaced fractures, open fractures and septal hematomas. ${ }^{14}$

A s trauma is the most common etiology, it can result in severe disruption of the nasal bones and cartilages resulting in significant external deformity and airway obstruction. ${ }^{15}$ The type and severity of nasal fracture is dependent on the force, direction and mechanism of injury. ${ }^{15} \mathrm{~L}$ ateral nasal trauma is most common and may result in the fracture of one or both nasal bones and sometimes dislocation of the nasal septum off the maxillary crest. ${ }^{15}$

Septal dislocation usually causes airway obstruction. ${ }^{15}$ Direct frontal trauma to the nose often results in the depression and widening of the nasal dorsum with associated nasal obstruction. ${ }^{15} \mathrm{M}$ ore severe injuries may result in comminution of the entire nasal pyramid. ${ }^{15}$ If these fractures are not properly diagnosed and corrected, the patient will have a poor cosmetic and functional outcome. ${ }^{15}$

The advantages of reduction and splinting under local anesthesia over general anesthesia include (1) shortened hospital stay, (2) ability of the patient to see cosmetic appearance after manipulation while still under anesthesia allowing further adjustment, (3) minimal delay between diagnosis and treatment, (4) reduction in operative costs, (5) elimination of general anesthesia risks, (6) option of general anesthesia available if local fails. ${ }^{16,17}$

Bone healing may occur more rapidly in the pediatric population, and so splinting for two weeks is enough. ${ }^{6}$ Four osteotomies were done with external rhinoplasty approach, and so POP cast was appropriate for them. Ten cases underwent augmentation rhinoplasty under internal approach and less bulky aluminium splints were used. More severe injuries, such as open fractures, septal hematoma, and injuries with gross external deformity require immediate surgical intervention reduction and $\mathrm{POP}$ splinting. ${ }^{6}$

\section{CONCLUSION}

Plaster of Paris splints give the best stabilization for nasal bone fractures as well as for rhinoplasties but are bulky and conspicuous. Aluminium nasal splints are not bulky and conspicuous but cannot be used for fractures with lacerations and external rhinoplasties. All fractures with edema should be managed once edema reduces, except in grossly displaced fractures, open fractures and septal hematomas.

Reduction and splinting of nasal bone fractures under local anesthesia is a safe and effective alternate to general anesthesia. $\mathrm{N}$ asal patency and cosmetic results are equally good as with procedure under general anesthesia. External rhinoplasties were splinted with POP and internal rhinoplasties by aluminium splints.

\section{REFERENCES}

1. Rohrich RJ, A dams WP (J r). Nasal fracture management: $M$ inimizing secondary nasal deformities. Plast. Reconstr Surg 2000;106:266-73. 
2. Drezner DA. Thermoplastic splint for use after nasal fracture. Otolaryngol. Head Neck Surg 1994;111:146-47.

3. A hn M S, M aas CS, M onhian N. A novel, conformable, rapidly setting nasal splint material: Results of a prospective study. A rch Facial Plast Surg 2003;5:189-92.

4. Wurtele P. Fixing external nasal splint with Tegaderm IV dressing J Otolaryngol 1990;19:79.

5. M atti BA, Nicolle FV. Use of Orthoplast as nasal splint. Br J Plast Surg 1986;39:414-16.

6. S Gunasekaran, M Jaramillo, Pothier DD. A Cost-Effective External Splint D evice for Nasal Fractures and R hinoplasty: A Technical Note. The Internet J ournal of Otorhinolaryngology 2006;4(2).

7. Hussain K, W ijetunge DB, Grubnic S, Jackson IT. A comprehensive analysis of craniofacial trauma. J Trauma 1994; 36:34-47.

8. Logan M, O'Driscoll K, M asterson J. The utility of nasal bone radiographs in nasal trauma. Clin Radiol 1994;49:192-94.

9. M uraoka M, Nakai Y, Shimada K, Nakaki Y . 10 year statistics and observations of facial bone fractures. A cta O tolaryngol Suppl 1991;486:217-23.
10. Scherer M, Sullivan WG, Smith DJ, Philips LG, Robson M C. A $n$ analysis of 1423 facial fractures in 788 patients at an urban trauma center. J Trauma 1989;29:388-90.

11. M urray J A, M aran A G, M ackenzie IJ, Raab G. Open vs closed reduction of the fractured nose. A rch Otolaryngol 1984;110: 797-802.

12. Chadha NK, Repanos C, Carswell AJ. Local anaesthesia for manipulation of nasal fractures: Systematic review. The J ournal of L aryngology and Otology 2009;123:830-36.

13. Illum P. L ong-term results after treatment of nasal fractures. J Laryngol Otol 1986;100:273-77.

14. Brian Rubinstein, E Bradley Strong. M anagement of Nasal Fractures. A rch Fam M ed 2000;9:738-42.

15. Colton J, Beekhuis G. M anagement of nasal fractures. Otolaryngol Clin N orth A m 1986;19:73-85.

16. Cook J, M cRae D, Irving R, D owie L. A randomized comparison of manipulation of the fractured nose under local and general anaesthesia. Clin Otolaryngol 1990;15:343-46.

17. W aldron J, M itchell DB, Ford G. Reduction of fractured nasal bones: L ocal versus general anaesthesia. Clin O tolaryngol 1989; 14:357-59. 